\title{
Habilidades funcionais e qualidade de vida em crianças com leucemia linfoblástica aguda
}

\author{
Functional abilities and quality of life in children with acute lymphoblastic \\ leukemia
}

\section{(iD) Meyene Duque Weber ${ }^{1}$ \\ (iD) Leila Simone Foerster Merey ${ }^{2}$ \\ (iD) Daniele de Almeida Soares-Marangoni ${ }^{3}$}

Parecer de Aprovação do Comitê de Ética em Pesquisa em Seres Humanos da Universidade Federal de Mato Grosso do Sul no. 2.407.274, 2017 .

Endereço para correspondência

Meyene Duque Weber.

Avenida das Gardênias, 185, Apto 21, Cidade Jardim, CEP: 13566-540, São Carlos-SP, Brasil.

+55 (67) 9.8183-6370

meyeneweber.nenem@gmail.com

\section{Resumo}

Objetivo: Comparar as capacidades funcionais e a qualidade de vida entre crianças com leucemia linfoblástica aguda em tratamento com quimioterapia e/ou radioterapia e crianças sem o tratamento, de 4 a 7 anos de idade.

Método: Participaram do estudo 50 crianças, sendo 25 em tratamento (grupo experimental) e 25 que já terminaram o tratamento (grupo controle), avaliados por meio do Inventário de Avaliação Pediátrica de Incapacidade (PEDI) e da Escala de Qualidade de Vida da Criança (AUQEI).

Resultados: Não houve diferença entre os grupos nos escores brutos do PEDI e na pontuação do AUQEI. Os escores normativos do PEDI apresentaram-se predominantemente abaixo do esperado para a idade em ambos os grupos. A pontuação do AUQEI demonstrou uma qualidade de vida prejudicada no grupo experimental e no controle.

Conclusão: Sugere-se que a leucemia linfoblástica aguda e seus tratamentos (quimio e radioterápicos) geram prejuízos funcionais e psicossociais, tanto no momento do tratamento oncológico como após sua conclusão e remissão da doença. É relevante que haja promoção de um acompanhamento longitudinal e multiprofissional da criança com câncer, a fim de minimizar os danos e reinseri-la na comunidade efetivamente.

Palavras-chaves: Leucemia. Criança. Qualidade de vida.

Abstract

Objective: To compare functional abilities and quality of life among children with acute lymphoblastic leukemia undergoing chemotherapy and/or radiotherapy and untreated children aged 4 to 7 years.

Method: 50 children, 25 in treatment (experimental group) and 25 without treatment (control group), were evaluated through the Pediatric Evaluation of Disability Inventory (PEDI) and the Quality of Life Evaluation Scale (AUQEI).

Results: There was no difference between the groups in the PEDI scores and the AUQEI score. PEDI normative scores were predominantly lower than expected for age in both groups. The AUQEI score showed an impaired quality of life in the experimental and control groups.

Conclusions: It is suggested that acute lymphoblastic leukemia and its treatments (chemo and radiotherapeutics) generate functional and psychosocial damages, both at the time of cancer treatment and after its completion and remission of the disease. It is relevant that there is promotion of a longitudinal and multiprofessional follow-up of the child with cancer, to minimize damages and reinsert it in the community effectively.

Keywords: Leukemia. Child. Quality of life.

\section{Cite como}

Vancouver

Weber, MD, Merey, LSF, Soares-Marangoni, DA. Habilidades funcionais e qualidade de vida em crianças com Leucemia Linfoblástica Aguda. Conscientiae Saúde 2020;19(1):1-13, e17042. https://doi.org/10.5585/conssaude.v19n1.17042. 


\section{Introdução}

O câncer infantil é considerado um problema de saúde pública e a principal causa de morte por doenças na população infanto-juvenil (1-19 anos) nos países em desenvolvimento1. A leucemia linfoblástica aguda (LLA), em particular, corresponde ao câncer com maior ocorrência nessa faixa etária, constituindo $85 \%$ das leucemias infantis e tendo seu pico de incidência entre os 2 e 5 anos de idade 2 . A leucemia é definida como uma patologia maligna dos leucócitos de etiologia desconhecida, e caracteriza-se pela substituição de células normais por células imaturas anormais na medula óssea ${ }^{3}$.

Com a melhoria e aprimoramento do tratamento em oncologia pediátrica, a taxa de sobrevivência aumentou significativamente. Apesar disso, o câncer infantil está associado a uma variedade de efeitos secundários à doença e aos tratamentos ${ }^{4}$. Enquanto comprometimentos musculoesqueléticos podem ser a manifestação mais primitiva da doença ${ }^{2}$, os tratamentos de quimioterapia e radioterapia são protocolos que frequentemente prejudicam o sistema neurológico e motor ${ }^{5-7}$.

A quimioterapia é uma estratégia que faz uso de compostos químicos (quimioterápicos) que afetam o funcionamento tanto de células neoplásicas quanto normais ${ }^{8}$. Desta forma, as crianças em tratamento tornam-se propensas a desenvolver diversos déficits motores, que terão impacto direto no seu desempenho funcional ${ }^{6}$. O tratamento radioterápico, por sua vez, consiste na impregnação de feixes de radiação ionizante capazes de suprimir as células tumorais, embora também possa danificar células normais circunvizinhas ${ }^{8}$. Também pode gerar perda extensa de tecido nervoso central ${ }^{5}$, quando há necessidade de irradiar todo o corpo ou tratar disseminações para o cérebro ou líquido espinhal. Por isso, a criança em radioterapia pode sofrer perda importante em seu repertório motor e ter sua recuperação funcional comprometida.

De forma geral, as crianças em tratamento de câncer podem apresentar limitações das habilidades motoras, o que dificulta ou impede a capacidade de participar das atividades sociais e físicas de seu dia-a-dia ${ }^{9}$. Consequentemente, é bastante possível que os principais efeitos adversos aos tratamentos quimio- e radioterápicos repercutam no bem estar biopsicossocial da criança com LLA, acarretando uma diminuição não só imediata, mas em médio ou longo prazos na sua qualidade de vida.

Apesar de ser um câncer comum na infância, há uma escassez de pesquisas que abordem seu impacto na funcionalidade e na qualidade de vida das crianças. Mesmo os estudos já realizados não abordam a funcionalidade em si, somente alguns aspectos motores associados, como equilíbrio, força, coordenação motora, entre outros. Além disso, esses estudos não 
abordaram uma faixa etária bem delimitada ou a LLA como tipo de câncer 6 ,10,11,12,13. Da mesma forma, em relação à análise da qualidade de vida, apesar dos estudos serem mais frequentes, abordam uma faixa etária abrangente e nem sempre são específicos quanto ao tipo de câncer estudado ${ }^{14-18}$.Sendo assim, ainda pouco se sabe sobre o impacto da LLA e das suas principais formas de tratamento (quimio e radioterapia) na funcionalidade e na qualidade de vida de crianças. Além disso, seria importante verificar esses aspectos em faixas etárias menores, quando várias habilidades motoras ainda estão se aperfeiçoando.

Considerando o contexto exposto, o objetivo do presente estudo foi comparar as capacidades funcionais e a qualidade de vida entre crianças com LLA em tratamento com quimioterapia e/ou radioterapia e crianças que já terminaram o tratamento, de 4 a 7 anos de idade. Este estudo pode se tornar uma importante contribuição para direcionar a promoção de saúde biopsicossocial e a reabilitação funcional e psicológica adequada para essa população.

\section{Materiais e Métodos}

\section{Desenho e Participantes}

Trata-se de um estudo transversal comparativo. As crianças foram recrutadas e avaliadas no Ambulatório e no Centro de Tratamento de Onco-Hematológico Infantil (CETOHI) do Hospital Regional de Mato Grosso do Sul (HRMS). Foram avaliadas 50 crianças com LLA, de ambos os sexos, com idade entre 4 e 7 anos, sendo 25 crianças em tratamento quimio e/ou radioterápico (grupo experimental) e 25 que já terminaram o tratamento (grupo controle).

Foram incluídas somente as crianças que realizavam o acompanhamento e/ou tratamento ambulatorial. Não foram incluídas crianças com diagnóstico clínico de distúrbios osteomioarticulares ou cognitivos associados.

O estudo foi aprovado pelo Comitê de Ética em Pesquisa em Seres Humanos da UFMS (parecer no. 2.407.274, 2017). Os pais e/ou responsáveis autorizaram a participação das crianças no estudo através da assinatura do Termo de Consentimento Livre e Esclarecido, assim como as crianças tiveram livre direito à escolha através do Termo de Assentimento.

\section{Instrumentos de Avaliação}

Os instrumentos de avaliação utilizados no estudo foram o Inventário de Avaliação Pediátrica de Incapacidade (PEDI) ${ }^{19}$ e a Escala de Qualidade de Vida da Criança (AUQEI) ${ }^{20}$.

O PEDI é um instrumento traduzido, adaptado e validado para o Brasil por Mancini $(2005)^{21}$. Tem objetivo de avaliar aspectos funcionais do desenvolvimento da criança de 6 
meses a 7 anos e 6 meses de idade. O questionário pode ser aplicado através da observação direta durante a execução das tarefas, por julgamento clínico ou de entrevista com o cuidador. O teste é composto por 3 partes. A parte I é destinada ao retrato da funcionalidade da criança em ambiente doméstico, referindo-se ao cumprimento de tarefas e atividades cotidianas em três áreas: autocuidado, mobilidade e função social. Para cada item, é atribuído escore 1 se a criança for apta à executar a atividade, ou escore 0 se não for capaz. A parte II expressa a quantidade de auxílio proporcionado pelo cuidador nas mesmas áreas. Neste caso, cada item é pontuado em uma escala ordinal, que varia de 5 (se a tarefa for executada de forma independente pela criança) a 0 (se a criança necessitar de total assistência e apoio para a execução da tarefa). Por fim, a parte III verifica se a criança se utiliza de alguma modificação do ambiente doméstico com o intuito de facilitar a execução ou o desempenho de suas atividades. Não há escala quantitativa nessa parte ${ }^{21}$. No presente estudo foram utilizadas apenas as áreas de autocuidado e mobilidade das partes I e II, sendo considerado o escore bruto. Quanto maior o escore, melhor o desempenho da criança nas habilidades funcionais e maior sua independência nas atividades de vida diária (AVDs) em relação ao cuidador. O escore normativo foi utilizado para classificar o desempenho funcional das crianças na faixa etária estudada, sendo identificado a partir da transformação do escore total bruto baseado na padronização da escala. Uma vez obtido, é considerado normal entre 30 e 70 , sendo que abaixo de 30 é classificado como atraso ou desempenho significativamente inferior ao esperado para a idade, enquanto um escore acima de 70 corresponde a desempenho significativamente superior ao esperado ${ }^{21}$

O questionário AUQEI é um instrumento padronizado e validado para o Brasil por Assumpção Jr et al. (2000) ${ }^{22}$, para crianças de 4 a 12 anos. Possui o intuito de avaliar a sensação subjetiva de bem-estar e é composto de 26 questões abrangendo os domínios: autonomia, lazer, funções e família. A satisfação da criança é analisada a partir de 4 figuras (faces correspondentes aos estados "muito triste", "triste", "feliz" e "muito feliz") que são associadas a diversos domínios da vida, por meio de 26 questões que exploram relações familiares e sociais, atividades, saúde, funções corporais e separação. Para a quantificação dos resultados, são atribuídos valores de 0 a 3 para cada questão, sendo 0 para "muito triste", 1 para "triste", 2 para "feliz" e 3 para "muito feliz". Os dados obtidos são pontuados através da soma das pontuações por item, sendo que essa soma pode variar de 0 a 78 . O ponto de corte é 48 , sendo os menores valores associados a uma qualidade de vida prejudicada e, os valores maiores ou iguais a 48 , a uma qualidade de vida satisfatória ${ }^{22}$. 


\section{Procedimentos}

Os dados pessoais da criança (nome, sexo, idade, data de nascimento, moradia e série escolar) e do responsável (nome, sexo, parentesco com a criança e escolaridade) foram coletados seguindo o formulário de pontuação inicial do PEDI.

As avaliações foram realizadas em um único momento, com uma duração total de, aproximadamente, 60 minutos. Os participantes do grupo controle eram avaliados na sala de espera do Ambulatório do HRMS e os do grupo experimental, na sala de quimioterapia ou na Brinquedoteca do CETOHI do HRMS.

O PEDI foi aplicado ao cuidador, no formato de entrevista. Posteriormente, o AUQEI foi aplicado à criança, finalizando a avaliação. Ambos os questionários foram empregados de acordo com as normas e exigências de cada um, assim como os resultados encontrados foram interpretados conforme as instruções e referências de cada instrumento.

\section{Variáveis e Análise Estatística}

A Capacidade Funcional e a Qualidade de Vida, mensuradas por meio do PEDI e do AUQEI, respectivamente, foram consideradas variáveis dependentes primárias.

A análise estatística foi realizada por meio do programa SPPS 23.0, respeitando-se os pressupostos de normalidade (Shapiro-Wilk) e homogeneidade (Levene). Para a capacidade funcional foram considerados os valores médios dos escores brutos das áreas de autocuidado e mobilidade nas Partes I e II do PEDI em cada grupo; para a qualidade de vida, foram considerados os valores médios da somatória de pontos em cada grupo. O teste de MannWhitney foi utilizado para comparar os grupos (tratamento x pós-tratamento) quanto ao AUQEI, e ANOVA One Way foi aplicada para comparação entre os grupos quanto ao PEDI. Foi adotado nível de significância $\alpha$ de $5 \%$ para as análises.

\section{Resultados}

\section{Características da amostra}

Não houve diferenças entre o grupo experimental e o grupo controle para as principais características da amostra, exceto para o tempo de interrupção do tratamento, maior no grupo controle (Tabela 1). 
Tabela 1 - Caracterização da amostra

\begin{tabular}{lccc}
\hline Características & GE & GC & $\boldsymbol{P}$ \\
\hline Idade (anos) & $5,68 \pm 1,10$ & $5,84 \pm 0,94$ & 0,585 \\
Escolaridade (anos de estudo) & $1,28 \pm 0,97$ & $1,24 \pm 0,59$ & 0,862 \\
Tempo de tratamento (anos) & $2,2 \pm 1,11$ & $2,72 \pm 1,20$ & 0,121 \\
Tempo de interrupção do tratamento (meses) & $*$ & $7,52 \pm 3,83$ & 0,000 \\
\hline
\end{tabular}

$\mathrm{M}=$ média; $\mathrm{DP}$ = desvio padrão; GE = grupo experimental; GC = grupo controle. *Não se aplica. Sexo: 13 meninas e 12 meninos no GC e 14 meninos e 11 meninas no GC.

Fonte: Própria autora.

\section{Qualidade de vida}

Não houve diferenças entre o grupo experimental e o grupo controle na pontuação do AUQEI. Ambos os grupos apresentaram qualidade de vida abaixo da pontuação de corte (Tabela 2).

Tabela 2 - Escores da Escala de Qualidade de Vida da Criança (AUQEI)

\begin{tabular}{lcccc}
\hline \multicolumn{1}{c}{ AUQEI } & $\begin{array}{c}\text { GE } \\
\mathbf{M} \pm \mathbf{D P}\end{array}$ & $\begin{array}{c}\mathbf{G C} \\
\mathbf{M} \pm \mathbf{D P}\end{array}$ & $\mathbf{U}$ & $\boldsymbol{p}$ \\
\hline Autonomia & $6,12 \pm 2,74$ & $7,52 \pm 3,61$ & 236,000 & 0,135 \\
Lazer & $12 \pm 3,31$ & $10,84 \pm 3,36$ & 242,000 & 0,168 \\
Função & $9,64 \pm 2,59$ & $10,88 \pm 2,60$ & 219,500 & 0,068 \\
Família & $10,04 \pm 3,22$ & $10,80 \pm 2,06$ & 250,5000 & 0,221 \\
Total & $37,58 \pm 6,56$ & $40,04 \pm 5,16$ & 243,000 & 0,175 \\
\hline
\end{tabular}

$\mathrm{GE}$ = grupo experimental; $\mathrm{GC}=$ grupo controle; $\mathrm{M}=$ média; $\mathrm{DP}=$ desvio padrão. Fonte: Própria autora.

\section{Desempenho funcional}

Não houve diferenças entre os grupos nos escores brutos do PEDI (Tabela 3; Figura 1). Ambos os grupos apresentaram predominantemente desempenho funcional atrasado considerando-se os escores normativos (faixa de normalidade entre 30 e 70) (Tabela 4). 
Tabela 3 - Escores brutos no Inventário de Avaliação Pediátrica de Incapacidade (PEDI)

\begin{tabular}{cccccc}
\hline \multicolumn{1}{c}{ Área do PEDI } & & $\begin{array}{c}\text { GE } \\
\text { M } \pm \mathbf{D P}\end{array}$ & $\begin{array}{c}\text { GC } \\
\text { M } \pm \mathbf{D P}\end{array}$ & $\mathbf{F}$ & $\boldsymbol{p}$ \\
\hline Habilidades funcionais & Autocuidado & $47,04 \pm 8,36$ & $44,60 \pm 8,29$ & 1,072 & 0,306 \\
& Mobilidade & $42,84 \pm 5,97$ & $45,56 \pm 7,63$ & 1,970 & 0,167 \\
Assistência do cuidador & Autocuidado & $31,84 \pm 4,73$ & $30,12 \pm 5,65$ & 1,359 & 0,249 \\
& Mobilidade & $29,44 \pm 3,99$ & $28,32 \pm 3,80$ & 1,032 & 0,315 \\
\hline
\end{tabular}

$\mathrm{GE}=$ grupo experimental; $\mathrm{GC}=$ grupo controle; $\mathrm{M}=$ média; $\mathrm{DP}=$ desvio padrão.

Fonte: Própria autora.

Figura 1 - Escores brutos médios no Inventário de Avaliação Pediátrica de Incapacidade (PEDI) por grupo

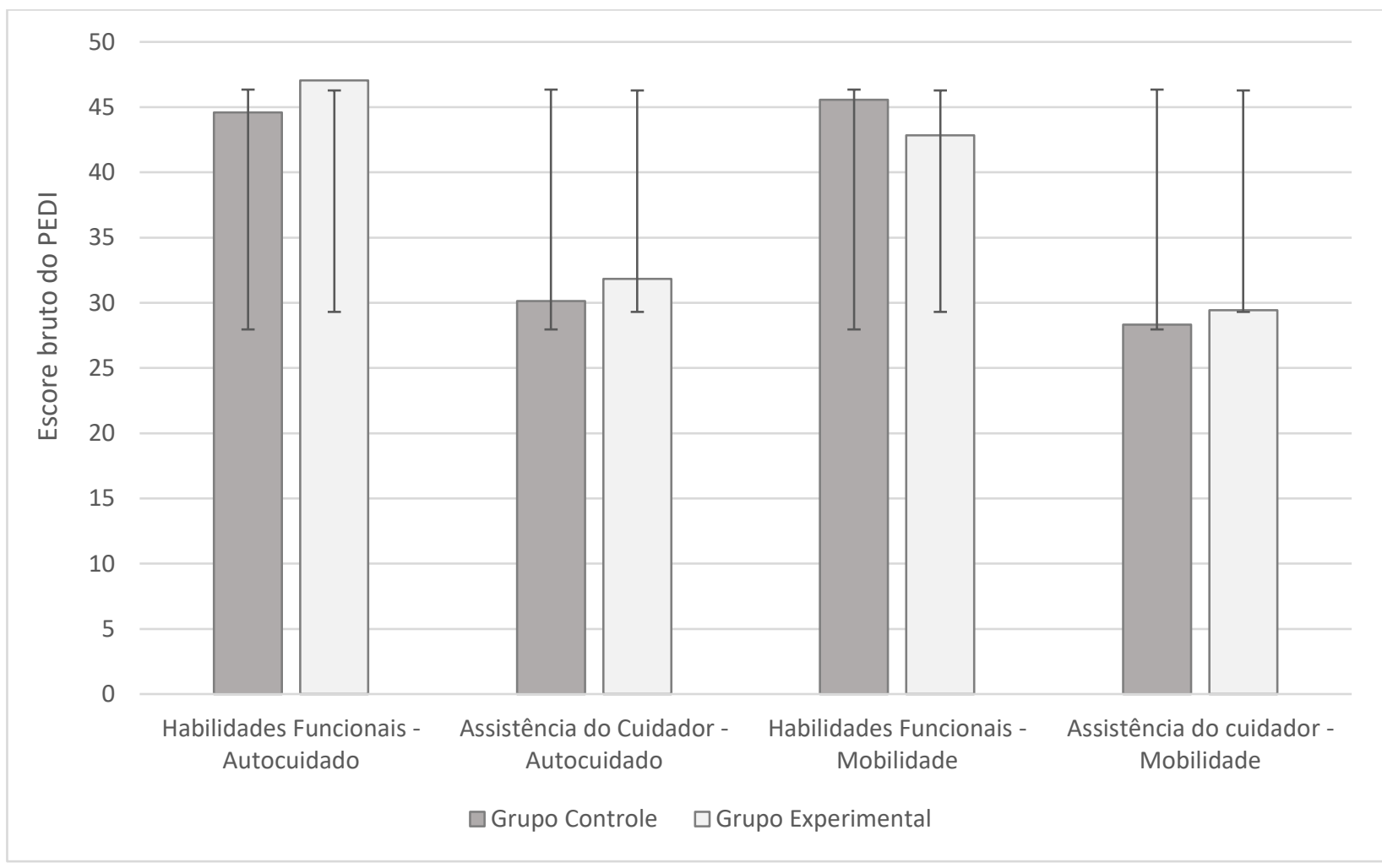

Fonte: Própria autora.

Tabela 4 - Escores normativos abaixo do esperado para idade no Inventário de Avaliação Pediátrica de Incapacidade (PEDI)

\begin{tabular}{cccc}
\hline \multicolumn{1}{c}{ Área do PEDI } & & GE & GC \\
& & $(\mathbf{N = 2 5 )}$ & $(\mathbf{N = 2 5 )}$ \\
\hline Habilidades funcionais & Autocuidado & $23(92 \%)$ & $25(100 \%)$ \\
& Mobilidade & $24(96 \%)$ & $22(88 \%)$ \\
Assistência do cuidador & $\begin{array}{c}\text { Autocuidado } \\
\text { Mobilidade }\end{array}$ & $5(20 \%)$ & $8(32 \%)$ \\
& & $19(76 \%)$ & $21(84 \%)$ \\
\hline
\end{tabular}

$\mathrm{GE}=$ grupo experimental; $\mathrm{GC}=$ grupo controle.

Fonte: Própria autora. 


\section{Discussão}

O presente estudo teve por objetivo comparar a capacidade funcional e a qualidade de vida entre crianças com LLA em tratamento com quimioterapia e/ou radioterapia e crianças sem o tratamento, de 4 a 7 anos de idade. Não foram encontradas diferenças entre os grupos, tanto na análise do AUQEI quanto do PEDI.

Primeiramente, é importante salientar que os grupos se apresentaram homogêneos em relação à idade, sexo, tempo de tratamento e escolaridade. Apenas o tempo de interrupção do tratamento divergiu deste padrão. Entretanto, este é um dado esperado, uma vez que apenas as crianças do grupo controle não estavam mais em tratamento. Estas informações indicam que tais fatores não influenciaram os resultados encontrados.

Não houve diferença entre os grupos na análise do PEDI. Entretanto, a similaridade dos escores entre os grupos, inclusive de resultados que expõem um desempenho funcional prejudicado, indicam a severidade do comprometimento gerado pela LLA e seu tratamento independentemente do tempo de interrupção do mesmo, desde que pelo menos a interrupção tenha ocorrido há menos de cerca de 11 meses. A literatura evidencia que a perda gradual da capacidade funcional, se dá por influência de sintomas como fadiga crônica e dor articular, que reduzem a prática de atividades de vida diária e afetam a mobilidade da criança ${ }^{23,24}$. A potencial neurotoxicidade das terapias antineoplásicas em conjunto com o atual estado maturacional do sistema nervoso do indivíduo, atingem alguns domínios como o funcionamento viso-motor e executivo e a velocidade de processamento de informações ${ }^{5}$. Alterações na força muscular e na flexibilidade, que acarretam déficits motores, também são advindas do tratamento farmacológico, como na prescrição de corticosteróides ${ }^{6}$. Nesse sentido, há relação com o futuro desenvolvimento cerebral da criança, visto que os efeitos tardios da doença e de seus tratamentos incluem o prejuízo da neuroplasticidade ${ }^{5}$.

Similarmente ao presente estudo, Hartman et al. (2013) ${ }^{13}$ encontraram a capacidade funcional reduzida em crianças entre 9 e 18 anos de idade que completaram o tratamento há mais de 5 anos, apesar de ter havido melhora da performance motora. Segundo os autores, não há uma maneira efetiva de distinguir se os comprometimentos em longo prazo se dão pela doença ou pelas formas de tratamento, sendo necessário um acompanhamento longitudinal para identificar os principais déficits manifestados por essa população. San Juan et al. (2008) ${ }^{25}$ identificaram a diminuição da funcionalidade global de crianças com LLA, de 4 a 12 anos de idade, em comparação a crianças saudáveis na mesma faixa etária. Em complemento, a revisão sistemática de Van Brussel et al. $(2005)^{26}$ verificou a aptidão física de crianças com LLA 
diminuída em todos os estudos em comparação a crianças saudáveis, de 7 a 19 anos de idade, refletindo diretamente na qualidade de vida e nos níveis de fadiga e, consequentemente, afetando a funcionalidade global da criança.

Também não houve diferenças entre os grupos na avaliação da qualidade de vida pelo AUQEI. O desempenho no questionário AUQEI, no entanto, indica uma qualidade de vida prejudicada em ambos os grupos. Isto sugere que mesmo sendo crianças em diferentes estágios da LLA, os efeitos causados por ela e por seus tratamentos geraram prejuízos ao bem estar biopsicossocial, o que naturalmente afeta a qualidade de vida. Os domínios contidos no AUQEI permitem obter um perfil de satisfação da criança a partir de diversas situações. As questões relativas a independência e relações interpessoais são representadas pelo item "autonomia". O atual cenário social não empodera a criança como protagonista de seus interesses, e tal contexto é reforçado diante de uma situação de adoecimento ${ }^{22}$. Tal domínio apresentou-se com a menor pontuação, evidenciando o impacto dessa imposição familiar como mecanismo protetivo, na vida da criança. As demais questões, como as relativas às férias, às brincadeiras e às relações familiares, possuem uma relação importante com a rotina médica e de proximidade com os pais, e compõem os domínios "lazer", "função" e "família". Bezerrra, Oliveira e Maia (2016) 27 identificaram que a redução da qualidade de vida, mensurada pelo AUQEI, se correlaciona ao estresse em crianças hospitalizadas por doença renal crônica, com idade entre 6 e 12 anos. Uma vez que crianças com LLA possuem alterações no desempenho funcional e são submetidas constantemente a exames, consultas médicas e possivelmente sofrem apreensão pessoal e familiar, acreditamos que no presente estudo tal rotina tenha contribuído no prejuízo da qualidade de vida. Vetsch et al. (2018) ${ }^{28}$, em sua revisão sistemática identificaram, em grande parte dos estudos analisados, uma qualidade de vida prejudicada em sobreviventes da LLA de idade variável (5 a 54 anos) quando comparados a indivíduos saudáveis, principalmente nos domínios psicossociais. Em contrapartida, Aznar et al. $(2018)^{29}$ verificaram uma melhor qualidade de vida em adultos sobreviventes da LLA quando comparados à população geral, utilizando como instrumento de avaliação o questionário SF-36 (36-Item Short Form Health Survey), que, diferente do AUQEI, não é voltado para a população pediátrica e, portanto, abrange domínios mais complexos. Tais indivíduos apresentaram alta prevalência de comorbidades crônicas associadas, que são advindas do tratamento oncológico recebido. No entanto, a percepção de qualidade de vida foi avaliada de maneira positiva, sobressaindo-se em relação a média da população geral ${ }^{29}$. Em suma, a similaridade dos resultados entre os grupos no presente estudo aponta a significância da repercussão física e psicológica gerada durante todo o processo de reabilitação oncológica em crianças com LLA, mesmo alguns meses após a 
interrupção do tratamento. Ao avaliar a qualidade de vida de pacientes oncológicos, é necessário considerar a subjetividade de seus sintomas, sejam esses físicos ou psicológicos, e o impacto destes no bem-estar do indivíduo, considerando que a percepção de saúde se dá de maneira particular, e é construída de acordo com as experiências pessoais do indivíduo ${ }^{28}$.

É importante enfatizar que o fato de a avaliação das crianças ter sido pontual é uma limitação deste estudo. Apesar disso, a análise pontual com o tempo de tratamento variável, realizada no presente estudo, permite uma visão ampliada do perfil dessas crianças e pode servir de base para uma avaliação integral da criança oncológica. Um estudo futuro com acompanhamento longitudinal, do momento em que o tratamento é iniciado até sua finalização, possibilitará identificar com maior consistência o desempenho funcional e a possível variação da performance durante esse período.

Por fim, deve-se ressaltar a importância de estudos que comparem a funcionalidade e a qualidade de vida em crianças durante e após o tratamento oncológico, utilizando-se de diversas ferramentas avaliativas para se atestar as possibilidades de comprometimento, analisando a criança de forma mais integral e, principalmente, em suas particularidades. O acompanhamento multidisciplinar é uma ferramenta necessária para minimizar os efeitos do tratamento oncológico em curto e longo prazos.

\section{Conclusão}

Em conclusão, o desempenho funcional e a qualidade de vida não expressaram diferença significativa quando comparados entre crianças com LLA em tratamento com quimioterapia e/ou radioterapia e crianças sem o tratamento na faixa etária de 4 a 7 anos de idade. No entanto, todas as crianças apresentaram-se prejudicadas nesses desfechos. Sugere-se que isso se deve aos prejuízos funcionais e psicossociais advindos da LLA e de seus tratamentos (quimio e radioterápicos), seja no momento do tratamento oncológico ou após sua conclusão e a remissão da doença.

\section{Agradecimentos}

Agradecemos à equipe do Hospital Regional de Mato Grosso do Sul, em especial ao Centro de Tratamento de Onco-Hematológico Infantil e ao Ambulatório, por toda atenção e colaboração. Às crianças e famílias voluntárias, o nosso agradecimento pela gentileza e pelo zelo ao colaborar para o desenvolvimento deste estudo. 


\section{Referências}

1. Instituto Nacional de Câncer (Brasil) [homepage na internet]. Câncer na criança e no adolescente no brasil: dados dos registros de base populacional e de mortalidade. Rio de Janeiro: INCA; 2008 [acesso em 27 maio 2018]. Disponível em:

http://www.inca.gov.br/tumores_infantis/pdf/livro_tumores_infantis_0904.pdf.

\section{Barbosa CMPL, Nakamura C, Terreri MT, Lee MLM, Petrilli AS, Hilário MOE.}

Manifestações músculo-esqueléticas como apresentação inicial das leucemias agudas na infância. J. Pediatr 2002;78(6):481-4.

3. Instituto Nacional de Câncer (Brasil) [homepage na internet]. Tipos de Câncer. Rio de Janeiro: INCA [acesso em 27 maio 2018]. Rio de Janeiro: INCA; Disponível em: http://www2.inca.gov.br/wps/wcm/connect/tiposdecancer/site/home.

4. Baumann FT, Bloch W, Beulertz J. Clinical exercise interventions in pediatric oncology: a systematic review. Pediatr Res 2013;74(4):366-74.

5. Hazin I, Garcia D, Gomes E, Leite D, Balaban B, Guerra A, et al. Desempenho intelectual pós tratamento de câncer: um estudo com crianças. Psicol Reflex Crit 2015;28(3):565-73.

6. Varedi M, Mckenna R, Lamberg EM. Balance in children with acute lymphoblastic leukemia. Pediatr Int 2017;59(3)293-302.

7. Molinari PCC, Lederman HM, Lee MLM, Caran EMM. Avaliação dos efeitos ósseos tardios e composição corporal de crianças e adolescentes tratados de leucemia linfoide aguda segundo protocolos brasileiros. Rev Paul Pediatr 2017;35(1):78-85.

8. Instituto Nacional de Câncer (Brasil) [homepage na internet]. Tratamento do Câncer. Rio de Janeiro: INCA [acesso em 28 maio 2018]. Disponível em:

http://www2.inca.gov.br/wps/wcm/connect/cancer/site/tratamento.

9. de Sá Rodrigues K, de Oliveira B, Borato Viana M. Aspectos da qualidade de vida relacionada à saúde em oncologia pediátrica. Rev Med Minas Gerais 2009;19: 42-47.

10. Götte M, Kesting SV, Winter CC, Rosenbaum D, Boos J. Motor performance in children and adolescents with cancer at the end of acute treatment phase. Eur J Pediatr 2015;174(6):791-9.

11. Tan SY, Poh BK, Chong HX, Ismail MN, Rahman J, Zarina AL, et al. Physical activity of pediatric patients with acute leukemia undergoing induction or consolidation chemotherapy. Leuk Res 2013;37(1):14-20.

12. Knight S, McCarthy M, Anderson V, Hutchinson E, De Luca C. Visuomotor function in children treated for acute lymphoblastic leukaemia with chemotherapy only. Dev

Neuropsychol 2014;39(2):101-12.

13. Hartman A, Hop W, Takken T, Pieters R, Heuvel-Eibrink M. Motor performance and functional exercise capacity in survivors of pediatric acute lymphoblastic leukemia. Pediatr Blood Cancer 2013;60(3):494-9. 
14. Queiroz DM, Amorim MH, Zandonade E, Miotto MH. Quality of life of children and adolescents with cancer: revision of studies literature that used the Pediatric Quality of Life Inventory. Invest Educ Enferm 2015;33(2):343-54

15. Batalha LMC, Fernandes AM, Campos C. Quality of life among children with cancer: agreement between child and parent reports. Esc Anna Nery 2015;19(2):292-6.

16. Lemos FA, Dal Bosco A, Sales VF, Silva SC, Dias AS. Análise de fatores físicos, motores e psicossociais em crianças com câncer. Rev AMRIGS 2013;57(2):95-100.

17. Dorantes-Acosta E, Villasís-Keever M, Zapata-Tarrés M, Arias-Gómez J, EscamillaNúñez A, Miranda-Lora A, et al. Calidad de vida de niños mexicanos con leucemia linfoblástica aguda atendidos por el Seguro Popular. Bol Med Hosp Infant Mex 2012;69(3):242-254.

18. Al-Gamal E, Long T. Health-related quality of life and its association with self-esteem and fatigue among children diagnosed with cancer. J Clin Nurs 2016;25(21-22):3391-9.

19. Haley S M, Coster WJ, Ludlow LH, Haltiwanger JT, Andrellos PJ. Pediatric evaluation of disability inventory (PEDI) version 1.0: development, standardization, and administration manual. Boston: Trustees of Boston University, Health and Disability Research Institute; 1992.

20. Manificat S, Dazord A. Évaluation de la qualité de vie de l'enfant: validation d'un questionnaire, premiers résultats. Neuropsychiatr Enfance Adolesc 1997;45(3):106-14.

21. Mancini, M. C. Inventário de avaliação pediátrica de incapacidade (PEDI): manual da versão brasileira adaptada. Belo Horizonte: UFMG, 2005.

22. Assumpção Jr, Francisco B, Kuczynski E, Sprovieri ME, Aranha EMG. Escala de avaliação de qualidade de vida: (AUQEI - Autoquestionnaire Qualité de Vie Enfant Imagé) validade e confiabilidade de uma escala para qualidade de vida em crianças de 4 a 12 anos. Arq. Neuro-Psiquiatr 2000;58(1):119-27.

23. Nunes MDR, Silva MCM, Rocha EL, Lima RAG, Nascimento LC. Measurement of fatigue in children and adolescents with cancer: an integrative review. Texto \& contexto enferm 2014;23(2): 492-501.

24. Shiari R, Salar N, Parvneh VJ, Rahmani K, Yeganeh MH, Shiari S. Midfoot Arthritis in Children: Is There Any Relation With Malignancy? Clin Med Insights Arthritis Musculoskelet Disord 2020;13:1179544120924643.

25. San Juan AF, Chamorro-Viña C, Maté-Muñoz JL, Fernández del Valle M, Cardona C, Hernández M, et al. Functional capacity of children with leukemia. Int J Sports Med 2008;29(2):163-7.

26. Van Brussel M, Takken T, Lucia A, Van Der Net J, Helders PJM. Is physical fitness decreased in survivors of childhood leukemia? a systematic review. Leukemia 2005;19(1):1317. 
27. Bezerra JC, Oliveira LCB, Maia EMC. Stress and quality of life in children with chronic kidney disease hospitalized. Psic Saúde \& Doença 2016;17(3):382-8.

28. Vetsch J, Wakefield CE, Robertson EG, Trahair TN, Mateos MK, Grootenhuis M, et al. Health-related quality of life of survivors of childhood acute lymphoblastic leukemia: a systematic review. Qual Life Res 2018;27(6):1431-33.

29. Aznar EGC, Casas AA, Bañeres AC, Escribano MACC, Aizpún JIL, Villagrasa PS. Calidad de vida y estados crónicos de salud en supervivientes de leucemia aguda infantil. Med Clín 2019;152(5):167-173. 